\title{
Análise do Internato em Medicina da Família e Comunidade de uma Universidade Pública de Fortaleza-CE na Perspectiva do Discente
}

\author{
Analysis of a Family Practice Internship Program at a Public \\ University in Fortaleza, Ceará, from the Student's Perspective
}

\author{
PALAVRAS-CHAVE \\ - Formação Médica. \\ - Medicina de Família e Comunidade. \\ - Internato e Residência. \\ - Atenção Primária à Saúde.
}

\author{
Ivana Cristina Vieira de Lima ${ }^{\mathrm{I}}$ (1) \\ Beatriz Yumi Rodrigues Shibuya ${ }^{1}($ D \\ Maria das Graças Barbosa Peixoto ${ }^{1}$ (iD \\ Leilson Lira de Lima ${ }^{1}$ (iD \\ Paulo Sávio Fontenele Magalhães ${ }^{1}($ iD
}

\section{RESUMO}

Introdução: A atuação do médico de familia e comunidade exige um domínio amplo de conhecimentos, habilidades e atitudes para enfrentar a complexidade dos pacientes, da familia e da comunidade, com vistas a agir para além da dimensão curativa. Nesse contexto, o internato consiste em uma experiência fundamental para promover a articulação teórico-prática da formação médica. Objetivou-se analisar o internato em Medicina de Família e Comunidade (MFC) de uma universidade pública de Fortaleza-CE, na perspectiva do discente. Métodos: Realizou-se estudo transversal, descritivo, quanti-qualitativo, em agosto e setembro de 2018 com 30 acadêmicos do curso de Medicina do $12^{\circ}$ semestre. A coleta de dados foi conduzida por meio de um questionário on-line que avaliou a percepção do acadêmico sobre a preceptoria, a aprendizagem, as aulas teóricas, a estrutura física das unidades, a satisfação geral com o internato e a relação com outros profissionais. Realizaram-se análise descritiva dos dados quantitativos e a análise de conteúdo do tipo temática. Resultados: A maioria dos acadêmicos era do sexo masculino (65,5\%) e estava na faixa etária de 22 a 24 anos (55,1\%). A satisfação geral com o internato foi considerada como boa (60\%) e as aulas teóricas também (63,3\%). O aprendizado foi avaliado como bom quanto à: relação médico-paciente e habilidades de comunicação (46,7\%); habilidade de registro em prontuário (33,3\%); habilidade para realizar atividades coletivas com usuários e equipe multiprofissional (43\%). Um total de $73 \%$ considerou que o internato contribuiu para a futura atuação profissional na atenção básica. Os aspectos positivos do internato foram: preceptoria; aprendizado acerca da relação médico-paciente; autonomia do discente na condução dos casos; carga horária para estudos; aproximação com a realidade da comunidade; inserção na rotina da unidade; diversidade na aprendizagem; e formação teórico-prática. Por sua vez, destacaram-se como aspectos negativos: infraestrutura da unidade básica de saúde; aprendizagem acerca de ações coletivas e interdisciplinaridade; realização de atividades essenciais; prática baseada em evidências; e preceptoria. Conclusão: De forma geral, o internato em MFC foi bem avaliado pelos discentes, principalmente quanto à preceptoria, ao aprendizado em habilidades clínicas e à contribuição para sua formação médica em uma futura atuação na atenção primária. São necessárias novas pesquisas avaliativas que envolvam a percepção dos preceptores e dos demais profissionais da equipe de saúde, com inclusão da análise da dimensão formativa. 


\section{KEYWORDS}

- Medical Training

- Family Practice.

- Internship and Residency.

- Primary Health Care.
ABSTRACT

Introduction: The work of a family medicine physician requires mastering a broad set of knowledge, skills and attitudes to deal with the complexity of patients, families and communities, with the aim of going beyond a healing perspective. In this context, the internship constitutes a fundamental experience to promote the theoretical and practical coordination of medical education. The objective of this study was to analyze a family practice internship program at a public university in the city of Fortaleza, state of Ceará, from the students' perspective. Methods: A cross-sectional, descriptive and quanti-qualitative study was developed in August and September of 2018 with 30 undergraduate medical students attending the $12^{\text {th }}$ semester of the course. Data were collected by means of an online questionnaire that assessed the students' perception on preceptorship, learning, theoretical classes, unit infrastructure, overall satisfaction with the internship program and their relationship with other professionals. Quantitative data were submitted to descriptive and thematic content analysis. Most undergraduate students were males, (65.5\%) aged between 22 and 24 years old (55.1\%). Results: Overall satisfaction with the internship was considered good (60\%), as well as with theoretical classes (63.3\%). Learning was assessed as good regarding the physician-patient relationship and communication skills (46.7\%); medical chart recording skills (33.3\%); skills to perform collective activities with users and multiprofessional teams (43\%). A total of $73 \%$ of students reported that the internship program contributed to their future professional performance in primary health care. The positive aspects of the internship program cited were: preceptorship; learning regarding the physician-patient relationship; students' autonomy in case conduction; course study load; getting closer to the reality of the communities; incorporation into the unit's routine; diversity in learning; and theoretical-practical training. The negative aspects were: infrastructure of the primary healthcare unit; learning of collective actions and interdisciplinarity; development of essential activities; evidence-based practice; and preceptorship. Conclusion: In general, the family practice internship program was well assessed by the students, mainly regarding preceptorship, learning clinical skills and the contribution to their medical education for future work in primary health care. Further assessment studies are needed, involving the perception of preceptors and other health professionals, and including an analysis of the educational dimension.

Recebido em 17/9/19

Aceito em 7/10/19

\section{INTRODUÇÃO}

Com o surgimento do Sistema Único de Saúde (SUS), a atenção primária à saúde (APS) foi estabelecida como estratégia de organização e integração do sistema, sendo então a Estratégia de Saúde da Família (ESF) considerada principal porta de entrada do sistema ${ }^{1}$. A implantação da ESF procurava reorganizar a atenção em saúde a fim de substituir o modelo tradicional. Iniciou-se, assim, a construção de um modelo de assistência baseado na promoção e proteção da saúde, no diagnóstico precoce e na recuperação dos agravos à saúde, de acordo com as diretrizes e os princípios do SUS $^{2-4}$.

Dessa forma, fortaleceu-se a crítica sobre o conceito saúde-doença e passou-se a considerar a necessidade de mudanças na formação dos profissionais da saúde, visto que a lei regulamentadora do SUS propunha a formação de profissionais com o perfil adequado aos seus princípios. Nesse momento, discutiu-se a importância da inserção de acadêmicos em projetos que promovessem a integração entre ensino, serviço e comunidade, valorizando a interdisplinaridade ${ }^{1-3}$.

A necessidade de aperfeiçoamento para suprir as carências da população nos níveis primários da atenção à saúde fez com que, em 1976, fosse criada a Medicina de Família e Comunidade (MFC), especialidade que se fundamenta em princípios que envolvem a atuação na APS, a atenção centrada na pessoa, a educação médica continuada, o humanismo e a formação de liderança. Trata-se, portanto, de uma área médica fundamental para o desenvolvimento da APS e consequentemente para a consolidação de um sistema de saúde resolutivo, abrangente e capaz de gerenciar de forma eficaz seus recursos ${ }^{1,5}$.

Assim, para atender às demandas sociais por saúde de qualidade e reformular a formação médica no Brasil, o Conselho Nacional de Educação instituiu, em 2001, as Diretrizes Curriculares Nacionais do Curso de Graduação em Medicina, que objetivam um egresso com formação generalista, humanista, crítica e reflexiva, que seja capaz a atuar, baseado em princípios éticos, no processo da saúde-doença, com ações de promoção, prevenção, recuperação e reabilitação à saúde, na perspectiva da integralidade da assistência, com senso de responsabilidade de social, como promotor da saúde integral do ser humano ${ }^{5,6}$. Para potencializar o alcance desses objetivos, é fundamental promover a integração entre ensino e serviço como um espaço de ensino-aprendizagem, oportunizando ao acadêmico a atuação comunitária e a apreensão das necessidades de saúde da população, seja em nível individual ou coletivo ${ }^{7}$.

Nesse contexto, as Diretrizes Curriculares Nacionais do Curso de Graduação em Medicina, instituídas a partir da Resolução no 3 em 2014, regulamentam como etapa integrante da graduação o estágio curricular obrigatório de formação em serviço, em regime de internato. Esse estágio deve destinar no mínimo de $30 \%$ da carga horária prevista para o internato médico da graduação em Medicina nas áreas de atenção básica e serviço de urgência e emergência do SUS, com prioridade para a APS ${ }^{8}$.

Evidências de estudos de avaliação do internato em APS realizados nas regiões Sul e Centro-Oeste do país indicam que a maioria dos

REVISTA BRASILEIRA DE EDUCAÇÃo MÉDICA

2 44 (1) : e006; 2020 
discentes considerou essa experiência como ótima ou boa ${ }^{6,9}$, além de classificá-la como bastante importante para promover maior segurança e autoconfiança no enfretamento das responsabilidades profissionais futuras $\mathrm{s}^{6,10,11}$.

Entre as dimensões mais bem avaliadas na perspectiva dos discentes, destacam-se: aulas teóricas; aprendizado em habilidades clínicas, comunicação e relação médico-paciente'; acompanhamento do preceptor; e relação preceptor-discente ${ }^{12}$. Por sua vez, a falta de estrutura física das unidades de saúde e a ausência de materiais/equipamentos são enfatizadas como aspectos negativos do internato em APS $^{6,13}$.

Tendo em vista a necessidade de uma formação médica integral e com foco na APS e considerando o internato como uma experiência fundamental para promover a articulação teórico-prática da formação médica, emergiram os seguintes questionamentos:

- Qual é o significado do internato em MFC para os discentes de uma universidade pública de Fortaleza-CE?

- Quais são os aspectos positivos e negativos da experiência do internato?

A importância desta pesquisa está na possibilidade de analisar o internato em MFC sob a perspectiva de discentes de uma instituição da Região Nordeste do país - onde há lacuna de estudos sobre essa abordagem -, bem como seus aspectos positivos e negativos, o que promoverá a apreensão da realidade vivenciada e a realização de possíveis mudanças para potencializar a experiência de integração teórico-prática no processo de ensino e aprendizagem no âmbito da APS. Além disso, o instrumento aplicado nesta pesquisa poderá ser incluído futuramente como uma avaliação formal do curso de Medicina da instituição investigada.

O objetivo da presente pesquisa foi analisar o internato em MFC de uma universidade pública de Fortaleza-CE, na perspectiva do discente.

\section{MÉTODO}

Trata-se de uma pesquisa descritiva, de abordagem quanti-qualitativa, realizada em agosto e setembro de 2018 em uma universidade pública de Fortaleza-CE. Em conformidade com as Diretrizes Curriculares Nacionais, os quatro últimos semestres da graduação nessa instituição de ensino se destinam à realização do estágio curricular obrigatório de treinamento em serviços, em regime de internato e sob supervisão de modo preceptoral. Essa etapa tem o objetivo de integrar os conhecimentos e as técnicas adquiridos nos semestres anteriores, com atividades eminentemente práticas de saúde individual e coletiva, em situações reais de exercício profissional. Os acadêmicos são distribuídos em sistema de rodízio em instituições de saúde das redes de atenção à saúde estadual e municipal, contemplando as seguintes áreas: clínica médica, clínica cirúrgica, pediatria, MFC, emergências Médicas e ginecologia e obstetrícia.

A população foi constituída por 39 acadêmicos do curso de Medicina da Universidade Estadual do Ceará (UECE), do $12^{\circ}$ semestre, que realizaram o internato de janeiro de 2017 a setembro de 2018 . Para a seleção dos acadêmicos do internato, utilizaram-se os seguintes critérios: 1. ser acadêmico de Medicina regularmente matriculado e 2. ter cursado o internato em MFC nos anos de 2017 e 2018, com finalização antes da coleta de dados da presente pesquisa. A amostra final foi composta por 30 acadêmicos.
A coleta de dados foi realizada por meio de um questionário on-line produzido na ferramenta Google Forms e enviado via e-mail pessoal dos acadêmicos, a partir de lista disponibilizada pela coordenação do curso de Medicina. Essa ferramenta permite a coleta de informações de forma simples e rápida, além de otimizar a análise dos dados da pesquisa ${ }^{14}$. Esse recurso foi escolhido em decorrência da dificuldade de agendar um momento individual com os acadêmicos, por causa da elevada carga horária semanal no período do internato, a qual foi evidenciada em pesquisa prévia realizada com o mesmo público-alvo ${ }^{15}$. Foi oferecido o prazo de 15 dias para os participantes responderem ao questionário.

O questionário é uma versão adaptada do instrumento utilizado no estudo de Silvestre, Tesser e Ros ${ }^{9}$, destinado a analisar a percepção do acadêmico de Medicina do internato sobre a preceptoria, a aprendizagem, as aulas teóricas durante o internato, a estrutura física das unidades de saúde, a satisfação geral com o internato e a relação com outros profissionais da APS. O questionário original é composto por 15 questões fechadas e quatro abertas, sendo as respostas à maioria das questões fechadas na forma de escala visual-analógica de faces - cinco faces categorizadas como ótima, boa, regular, ruim e péssima. O questionário também é constituído pelas seguintes questões abertas:

- Quais foram os pontos positivos e negativos do internato em MFC?

- Quais são as suas sugestões para a melhoria do internato em MFC?

A adaptação do questionário original consistiu na substituição da escala visual-analógica de faces pela escala de Likert e na adição de duas perguntas objetivas:

1) Assinale as atividades que você desenvolveu ao longo do internato em MFC: (opções de resposta: ações conjuntas com acadêmicos de outros cursos; acompanhamento de consultas com outros profissionais da equipe; acompanhamento de consultas médicas; anamnese centrada no paciente e exame físico; articular ou participar de ações intersetoriais de gestão; notificação compulsória; participar de reuniões da equipe de saúde; participar de atividades de promoção da saúde e organizálas; participar e/ou mobilizar atividades em participação com a comunidade; realizar referência e contrarreferência de pacientes; registro em prontuário; solicitação de exames; territorialização; visita domiciliar).

2) O aprendizado oferecido pelo internato em MFC em termos de conhecimento, habilidades e atitudes contribuiu para a sua futura atuação como médico na atenção primária à saúde? (opções de resposta: com certeza não; provavelmente não; provavelmente sim; com certeza sim; não sei).

Ressalta-se que, após a adaptação do questionário, ele foi submetido à análise pelos professores responsáveis pelo internato em MFC e aplicado a duas discentes que não compuseram a amostra, com o intuito de analisar a clareza das informações e a compreensão das perguntas.

Os dados foram organizados a partir de uma planilha do programa Microsoft Excel gerada automaticamente pela ferramenta Google Forms. Para as variáveis quantitativas, calcularam-se a frequência absoluta $e$ a relativa, além da média e do desvio padrão (DP) a partir do uso do software estatístico $\mathrm{R}$, versão 3.2.2. Criaram-se tabelas e quadros para otimizar a apresentação das informações.

REVISTA BRASILEIRA DE EDUCAÇ̄̃o MÉDICA

3 44 (1) : e006; 2020 
$\mathrm{O}$ conjunto de respostas às perguntas abertas do questionário foi reunido, lido e analisado a partir da análise de conteúdo do tipo temático. As seguintes etapas foram aplicadas para análise de conteúdo temático: pré-análise, exploração do material ou codificação, tratamento dos resultados, inferência e interpretação ${ }^{16}$. O registro das percepções emitidas por cada um dos discentes foi identificado pela letra " $D$ " (abreviação de discente), seguida por um algarismo arábico correspondente à ordem de resposta ao questionário.

A pesquisa faz parte de um estudo mais amplo, intitulado Avaliação da formação de profissionais de saúde de nível superior pelas universidades públicas em Fortaleza-CE, aprovado pelo Comitê de Ética em Pesquisa da UECE, sob o Parecer Consubstanciado no 388536/2013.

\section{RESULTADOS}

Entre os 30 respondentes, houve predominância de acadêmicos do sexo masculino $(65,5 \%)$ em faixa etária de 22 a 24 anos $(55,1 \%)$, com idade média de 24,4 (desvio padrão $=1,98$ ). Acerca da percepção geral sobre o internato em MFC, mais da metade classificou a satisfação geral como boa (60\%). Quanto às aulas teóricas durante o internato, 63,3\% avaliaram essa experiência como boa. Sobre o aprendizado referente à relação médico-paciente e habilidades de comunicação, um total de $46,7 \%$ classificou como bom e $43,3 \%$ como ótimo. No que se refere ao aprendizado quanto à habilidade de registro em prontuário, as respostas predominantes foram bom $(33,3 \%)$ ou regular $(33,3 \%)$. No que concerne ao aprendizado das habilidades para realizar atividades coletivas com os usuários e a equipe multiprofissional, a maior parte dos discentes considerou esse aprendizado como bom (43\%) ou regular (43\%).

No que se refere à percepção do discente sobre a contribuição do internato em MFC para uma futura atuação na APS, 73\% responderam que "com certeza" houve contribuição. Acerca da análise das expectativas em relação ao internato em MFC, metade dos acadêmicos mencionou que a experiência foi melhor do que imaginavam e apenas $13 \%$ referiram ter considerado a experiência pior que o esperado.

Acerca das atividades desenvolvidas durante o internato, as mais citadas foram: solicitação de exames (83\%), acompanhamento de consultas médicas (83\%), anamnese centrada no paciente/exame físico (83\%), visita domiciliar (83\%), registro em prontuário (80\%), notificação compulsória (56\%) e realização de referências para outros serviços ou profissionais da rede de atenção à saúde (56\%). Ressalta-se que algumas ações relativas à área de saúde coletiva foram desenvolvidas por um percentual menor de discentes, a saber: articular ações intersetoriais de gestão ou participar delas (23\%), participar de atividades de promoção da saúde e organizá-las (23\%), territorialização (26\%), acompanhamento de consulta com outros profissionais da equipe (33,3\%), participar de reuniões da equipe $(33,3 \%)$, participar de atividades com a comunidade e/ou mobilizá-las (40\%) e organizar atividades de educação em saúde $(43,3 \%)$ e ações conjuntas com acadêmicos de outros cursos (46\%) (Tabela 1).

Acerca dos aspectos positivos do internato, os depoimentos foram agrupados nas seguintes categorias: preceptoria; aprendizado acerca da relação médico-paciente; autonomia do discente na condução dos casos; carga horária para estudos; aproximação com a realidade da comunidade; inserção na rotina da unidade básica de saúde; diversidade na aprendizagem e formação teórico-prática. Por sua vez, as categorias temáticas relacionadas aos aspectos negativos do internato foram: infraestrutura da unidade básica de saúde; aprendizagem acerca de ações coletivas e interdisciplinaridade; realização de atividades essenciais; prática baseada em evidências; e preceptoria (Quadro 1).

\section{DISCUSSÃO}

Observou-se que a maioria dos discentes se considerou satisfeita quanto ao internato em MFC. Esse resultado é corroborado pelas respostas também positivas nas questões fechadas que avaliaram as aulas teóricas, o aprendizado sobre a relação médico-paciente/habilidades de comunicação, a aprendizagem quanto ao registro em prontuário e o aprendizado das habilidades para realizar atividades coletivas com usuários e equipe multiprofissional. No que se refere à comparação das expectativas, metade dos acadêmicos mencionaram que a experiência foi melhor do que imaginavam e apenas $13 \%$ referiram ter considerado a experiência pior que o esperado.

Nas questões abertas, fica evidente uma avaliação positiva do internato, a qual pode se relacionar a aspectos como acompanhamento satisfatório do preceptor; disponibilidade de tempo para o discente conciliar os estudos à rotina do internato; desenvolvimento da autonomia e de habilidades clínicas inerentes ao médico generalista, como mostram, por exemplo, as seguintes respostas: "Ótimos preceptores, compromissados, resolutivos"; "Tempo pra estudar e aprendizado constante"; "A autonomia do estudante em atender e atuar enquanto futuro profissional de saúde"; "Contato com grande variedade de especialidades/problemas em saúde”.

\begin{tabular}{|c|c|}
\hline $\begin{array}{c}\text { TABELA } 1 \\
\text { Atividades desenvolvidas no internato em MFC (n = }\end{array}$ & \\
\hline Atividade & $\mathrm{N}(\%)$ \\
\hline Territorialização & $8(26)$ \\
\hline Acompanhamento de consultas médicas & $25(83)$ \\
\hline Anamnese centrada no paciente e exame físico & $25(83)$ \\
\hline Solicitação de exames & $25(83)$ \\
\hline Visita domiciliar & $25(83)$ \\
\hline Registro em prontuário & $24(80)$ \\
\hline Notificação compulsória & $17(56)$ \\
\hline $\begin{array}{l}\text { Realização de referências para outros serviços ou } \\
\text { profissionais }\end{array}$ & $17(56)$ \\
\hline Ações conjuntas com acadêmicos de outros cursos & $14(46)$ \\
\hline $\begin{array}{l}\text { Participar de atividades de educação em saúde e } \\
\text { organizá-las }\end{array}$ & $13(43,3)$ \\
\hline $\begin{array}{l}\text { Participar e/ou mobilizar atividades em participação com } \\
\text { a comunidade }\end{array}$ & $12(40)$ \\
\hline $\begin{array}{l}\text { Acompanhamento de consultas com outros profissionais } \\
\text { da equipe }\end{array}$ & $10(33,3)$ \\
\hline Participação em reuniões da equipe de saúde & $10(33,3)$ \\
\hline Articular ações intersetoriais de gestão ou participar delas & $7(23)$ \\
\hline $\begin{array}{l}\text { Participar de atividades de promoção da saúde e } \\
\text { organizá-las }\end{array}$ & $7(23)$ \\
\hline
\end{tabular}

Fonte: Elaborada pelos autores. 


Aspectos positivos e negativos
Aspectos positivos
Preceptoria
"Ótimos preceptores, compromissados, resolutivos, e boa relação médico-
paciente" (D24).
"Preceptores preocupados com o ensino e interessados em nosso
conhecimento" (D6, D9).
"Boa relação com os preceptores, são muito solícitos em responder todas as
nossas dúvidas" (D5, D14).
"Relação professor-aluno horizontal" (D15, D21).
Aprendizagem acerca da relação médico-paciente
"Otimização da relação médico-paciente" (D5, D28).
"Aprender a relação médico-paciente" (D7, D16, D18).
“[...] treinar a relação médico-paciente para garantir a longitudinalidade do
atendimento" (D26).
atendimento" (D26).

\section{Autonomia do discente na condução dos casos}

"A autonomia do estudante em atender e atuar enquanto futuro profissional de saúde, com contato com situações clínicas do dia a dia" (D11).

"Independência para conduzir a consulta e apenas depois de finalizada a consulta passar o caso para o preceptor" (D20).

\section{Carga horária para estudos}

"Nesse serviço, existe carga horária mais adequada para conciliar os estudos necessários ao internato" (D6).

“Carga horária permite o estudo diário" (D19, D25).

"Tempo pra estudar e aprendizado constante" (D10).

\section{Aproximação com a realidade da comunidade}

"Visitas domiciliares e a áreas de risco com equipe multiprofissional" (D5).

"Conhecer a realidade (ambiente e cotidiano) da população atendida na unidade básica de saúde, através das visitas domiciliares” (D22).

"Pôr em prática os conhecimentos os conhecimentos sobre as patologias mais prevalentes de nossa comunidade" (D26).

Inserção na rotina da unidade básica de saúde

"Aprender sobre o funcionamento do posto de saúde e como abordar a saúde de uma comunidade" (D1, D18).

"Vivência da rotina de médico de família" (D5).

\section{Diversidade na aprendizagem}

"Ganho de experiência com pré-natal, puericultura e visita domiciliar” (D5, D19).

"Participação nas reuniões do conselho de saúde” (D5).

"Contato com grande variedade de especialidades/problemas em saúde" (D7, D17, D19).

"Participação em muitas atividades diferentes" (D18).

"Revisão do que vimos na prática e vemos patologias em vários níveis de severidade" (D29).

\section{Formação teórico-prática}

"Formação para atuação nas unidades básicas de saúde" (D2).

"A experiência adquirida no manejo da hipertensão arterial sistêmica e diabetes mellitus e das principais queixas na atenção primária” (A22).

"Organização adequada. Serviço voltado para ensino do aluno" (A23).

Fonte: Elaborada pelos autores.

Esses resultados vão ao encontro de pesquisas com abordagem semelhante realizadas nas regiões Sul e Centro-Oeste do país ${ }^{6,9,11}$, confirmando as vantagens desse tipo de experiência, na qual se promove um aprendizado baseado na vivência dos alunos, servindo como base para a análise de seus conhecimentos e aplicação na prática, a fim de formar um profissional capaz de tomar iniciativas e solucionar problemas ${ }^{12}$.
Preceptoria

"Limitada discussão sobre as patologias/queixas dos pacientes, além das condutas mais atuais" (D22).

"Pouca experiencia dos profissionais com MFC" (D25).

"Poucas atividades teóricas" (D17, D28).

\section{Aprendizagem acerca de ações coletivas e interdisciplinaridade}

"[...] queria ter tido a oportunidade de participar de reuniões de planejamento e gerenciamento do posto de saúde" (D1).

"Nos meus locais de estágio, não havia tanta interdisciplinaridade ou multidisciplinaridade" (D6).

"Não houve campanhas de promoção/educação em saúde” (D19).

\section{Realização de atividades essenciais}

"Em nenhum momento tive experiência em coleta de citologia oncótica, por exemplo" (D5).

"Não participei nenhum dia no setor de vacinação" (D5).

"Poucas visitas domiciliares" (D5).

"Boa parte dos estágios tive função de observar a consulta e ajudar o preceptor” (D6).

\section{Prática baseada em evidências}

"Senti falta de discussão dos casos baseada nos protocolos do Ministério da Saúde e das sociedades de cardiologia/endocrinologia etc." (D5).

"Em algumas situações, acompanhei condutas pouco adequadas do ponto de vista científico (condutas desatualizadas ou incorretas), embora essas fossem pouco frequentes" (D6).

\section{Infraestrutura da unidade básica de saúde}

"Infraestrutura deixa a desejar" (D2, D12).

"Os serviços de saúde não eram muito organizados, pacientes ficavam entrando no meio da consulta para pedir favores" (D3).

"Quantidade exagerada de demanda espontânea" (D5).

“O volume de pacientes que ocasionalmente é desproporcional" (D11).

"Dificuldade de resolução dos problemas dos pacientes" (D8).

O internato em MFC favorece a comunicação médico-paciente e promove maior habilidade, segurança e paciência na condução dos casos, ampliando a visão do discente quanto ao paciente como um ser biopsicossocial ${ }^{17}$. Assim, esse período de maior convívio com a comunidade permite ao aluno perceber a complexidade e a variedade dos fatores que determinam a qualidade de vida das famílias. 
Apesar de a preceptoria ter sido bem avaliada pela horizontalidade na relação com o discente e resolutividade, as questões abertas demonstram fragilidades nessa dimensão, no que tange à experiência dos preceptores em MFC e à metodologia de ensino-aprendizagem adotada, conforme observado nas seguintes respostas: "Pouca experiencia dos profissionais com MFC"; "Limitada discussão sobre as patologias/queixas dos pacientes, além das condutas mais atuais"; "Senti falta de discussão dos casos baseada nos protocolos do Ministério da Saúde".

A falta de experiência do preceptor em MFC aponta para a necessidade de expansão dos programas de residência nessa área, com vistas a fortalecer a formação médica generalista e a maior inserção de profissionais na APS ${ }^{18}$.

O papel do preceptor local merece destaque, visto ser um profissional que concilia os deveres e as obrigações do trabalho na ESF com o trabalho de inserir os discentes do internato, ser mediador entre a equipe de saúde, a secretaria municipal e o gestor local, com metas e objetivos de programas estabelecidos, sendo dele a responsabilidade pela condução desse processo complexo ${ }^{19}$. O docente torna-se assim um orientador no processo de construção da autonomia do aluno ${ }^{20}$. Ademais, o preceptor desempenha um papel essencial na formação médica, uma vez que o estudante se depara com o desafio de agregar conhecimentos da teoria à aplicação prática, de maneira segura e autônoma.

Se, por um lado, os discentes consideram o internato em APS uma ótima oportunidade para desenvolvimento da autonomia, um dos participantes, por outro, informou caráter passivo na aprendizagem, característico do modelo tradicional de ensino: "Boa parte dos estágios tive função de observar a consulta e ajudar o preceptor”. Destaca-se assim a necessidade de capacitar o professor de Medicina quanto ao exercício de seu papel como docente, a partir de cursos que o ajudem a potencializar o uso de estratégias pedagógicas no cenário de prática ${ }^{21}$.

Nesse contexto, a ampliação de discussões a partir de uma clínica baseada em evidências, com aulas mais interativas e mais estruturadas que proporcionem mais conhecimento prático, é considerada pelos discentes uma das ações importantes para a promover aprendizagem ativa durante o internato em MFC'.

A carência de embasamento teórico para a realização da prática pelos médicos preceptores, sem emprego de diretrizes clínicas, também foi referido em um estudo realizado em Minas Gerais ${ }^{21}$. Embora atualmente exista uma gama de recursos oferecidos aos profissionais de saúde para fundamentar a conduta clínica com base em evidências científicas, tanto para prevenção, diagnóstico e tratamento quanto para reabilitação, a utilização das diretrizes clínicas ainda consiste em um desafio em nível nacional ${ }^{22}$.

Apesar da importância da prática baseada em evidências na atenção à saúde, a sua implantação possui dificuldades nos serviços de saúde, como falta de estrutura física, de recursos humanos e de equipamentos, além da ausência de apoio da organização gerencial ${ }^{23}$. Assim, a dificuldade de efetivação dessa prática nos serviços de saúde depende também do contexto cultural da população, da carga de trabalho e dos administradores, e não apenas do profissional de saúde.

Além disso, mesmo que determinada conduta tenha alto grau de recomendação, é essencial que o profissional da saúde avalie sua adequação para uma realidade específica, adotando recomendações da literatura que contemplem o contexto local, a realidade socioeconômica e cultural. Dessa forma, as orientações clínicas e dos guidelines devem ser passíveis de adaptação à realidade da comunidade ${ }^{24,25}$.

Outros aspectos avaliados como positivos no internato em MFC foram a aproximação com a realidade da comunidade e a inserção na rotina da unidade básica de saúde, evidenciados nas respostas a seguir: "Conhecer a realidade da população atendida na unidade básica de saúde, através das visitas domiciliares"; "Vivência da rotina de médico de família".

O maior convívio com a comunidade, a participação em visitas e a integração com a equipe de saúde da família permitem ao aluno entender a complexidade de fatores que influenciam a qualidade de vida da população, compreendendo como suas atitudes podem promover a saúde do paciente e das famílias que ele acompanha. Dessa forma, o discente, além de tornar-se mais seguro em suas ações médicas, entende o paciente de forma holística e contextualizada ${ }^{17}$.

Ao inserir a prática médica do discente no ambiente do sistema de saúde, a escola médica cumpre seu papel em mudar as condições dos serviços de saúde oferecidos à comunidade, além de possibilitar uma aproximação com a realidade do mercado de trabalho e um aprendizado mais voltado para as necessidades de saúde da população ${ }^{26}$. Quando se aproxima o discente do treinamento profissional, desperta-se maior envolvimento cognitivo, pois isso o torna ativo na construção de seu conhecimento. Assim, quando se integra o treinamento do discente ao SUS, espaço onde os futuros médicos atuarão, pode-se alcançar o perfil almejado ${ }^{27}$.

Contudo, os aspectos negativos da aproximação do discente com a realidade do sistema de saúde também foram enfatizados em resposta às questões abertas: "Os serviços de saúde não eram muito organizados"; "Infraestrutura deixa a desejar"; "Quantidade exagerada de demanda espontânea"; "O volume de pacientes que ocasionalmente é desproporcional"; "Dificuldade de resolução dos problemas dos pacientes".

Problemas como a carência de infraestrutura nas unidades de saúde, com falta de materiais para realização de exame físico e de procedimentos, bem como de consultórios para os atendimentos, demonstram a precarização do sistema público de saúde $e^{6,13}$. Essa realidade repercute em atrasos e menor eficiência dos atendimentos aos pacientes, gerando desconforto e dificultando o ensino ${ }^{13}$. Isso demonstra a necessidade de que a infraestrutura do serviço seja adequada ao acolhimento do discente e do paciente, uma condição que não depende da universidade, mas sim de políticas mais amplas.

Dentre as atividades desenvolvidas durante o internato em MFC, a maior parte dos alunos realizou ações que envolveram o processo de atendimento do paciente, a saber: solicitação de exame, acompanhamento de consultas médicas, anamnese centrada no paciente e exame físico, visita domiciliar, registro em prontuário, notificação compulsória e realização de referências para outros serviços ou profissionais da rede de atenção à saúde.

Em convergência, nas respostas às questões abertas, há ênfase na diversidade da aprendizagem como aspecto positivo do internato, como demonstrado a seguir: "Ganho de experiência com pré-natal, puericultura e visita domiciliar"; "Participação em muitas atividades diferentes" "Revisão do que vimos na prática e vemos patologias em vários níveis de severidade”. Em contrapartida, foi mencionada ausência ou incipiência de experiências em atividades consideradas essenciais à formação médica: "Em nenhum momento tive experiência em coleta de citologia oncótica"; "Não participei nenhum dia no setor de vacinação"; "Poucas visitas domiciliares".

Evidências demonstram que os discentes valorizaram as práticas que não realizam comumente em nenhum outro serviço de saúde, tendo o

REVISTA BRASILEIRA DE EDUCAÇÃo MÉDICA

6 6 (1) : e006; 2020 
internato em MFC possibilitado que os alunos vivenciassem os princípios essenciais na APS, principalmente acessibilidade, longitudinalidade, trabalho em equipe e atuação na prevenção. Além disso, os acadêmicos consideraram que a participação em todas as atividades desenvolvidas foi o que possibilitou a real compreensão da $\mathrm{ESF}^{17}$.

As respostas às questões fechadas demonstraram ainda que os discentes tiveram menor oportunidade de participar ações coletivas e interdisciplinares, como: articular ações intersetoriais de gestão ou participar delas; participar de atividades de promoção da saúde e organizá-las; territorialização; acompanhamento de consulta com outros profissionais da equipe; participar de reuniões da equipe; participar de atividades com a comunidade e/ou mobilizá-las; participar de atividades de educação em saúde e organizálas; ações conjuntas com acadêmicos de outros cursos. Esses resultados são reafirmados nas respostas relativas aos aspectos negativos do internato nas questões abertas: “[...] queria ter tido a oportunidade de participar de reuniões de planejamento e gerenciamento do posto de saúde"; "Não houve campanhas de promoção/educação em saúde".

Apesar de a formação médica da universidade investigada oferecer disciplinas direcionadas ao desempenho de ações em âmbito coletivo, que contemplem a promoção de saúde, a prevenção de doenças, a recuperação e a reabilitação do indivíduo, ainda se evidencia na prática a dificuldade em romper as barreiras impostas pelo modelo biomédico. É imprescindível enfatizar o seguinte aspecto: por meio de ações como visitas, reuniões, atendimentos e da integração com a equipe da ESF, o estudante aprende como agir para promover a saúde do paciente e das famílias que ele acompanha ${ }^{17}$. Acredita-se que o melhor planejamento didático e capacitação dos preceptores possa gerar impactos positivos neste sentido.

\section{CONSIDERAÇÕES FINAIS}

O internato em MFC é reconhecido como uma experiência satisfatória, com repercussões positivas para o desenvolvimento da autonomia, a aproximação com a realidade da comunidade e a promoção da diversidade na aprendizagem. Recebeu qualificação positiva pela maioria dos participantes, na maior parte dos aspectos, com destaque para a preceptoria, o aprendizado em habilidades clínicas e a contribuição para sua formação médica em uma futura atuação na atenção primária.

$\mathrm{Na}$ perspectiva dos discentes, há a necessidade de capacitação dos preceptores quanto ao uso de metodologias de incentivo à autonomia do aluno, prática baseada em evidências e aprimoramento das atividades teóricas. $\mathrm{O}$ planejamento didático poderia ser implementado para maximizar o desenvolvimento de habilidades clínicas essenciais, bem como para promover a ampliação das ações coletivas e interdisciplinares.

Como limitações da presente pesquisa, destacam-se a carência de maiores informações sobre o perfil dos acadêmicos entrevistados, o caráter somativo da análise e o reduzido número de participantes, justificado pelo fato de a universidade formar discentes somente uma vez ao ano - fatores que limitam a generalização dos resultados. Sugere-se em pesquisas futuras uma avaliação mais abrangente do internato do ponto de vista dos preceptores e dos demais profissionais da equipe de saúde envolvidos no acompanhamento dos discentes, na qual sejam agregadas distintas metodologias, tais como a observação e a realização de avaliação formativa quanto às competências adquiridas pelos discentes, de maneira a efetuar mudanças enquanto o internato estiver em curso.

\section{REFERÊNCIAS}

1. Oliveira VG, Queiroz FN, Araújo BP, Silva CMM, Silva F. Medicina de Família e Comunidade: breve histórico, desafios e perspectivas na visão de discentes de graduação. Rev Bras Med Fam Comunidade 2014;9(30):85-8.

2. Xavier NF, Monteiro JCMS, Caldas CAM, Pires CAA. Pet-Saúde: o impacto do programa na formação do profissional médico. RBCS 2018;22(1):37-44.

3. Vieira RMM, Pinto TR, Melo LP. Narrativas e memórias de docentes médicos sobre o ensino baseado na comunidade no sertão nordestino. Rev Bras Educ Med 2018;42(1):142-51.

4. Melo BT, Cavalcante TM, Batista RSL, Jordão DA, Beserra KS, Andrade LSG et al. Uma experiência de integração ensino, serviço e comunidade de alunos do curso de graduação em Medicina na atenção básica no município de Maceió-AL, Brasil. Rev Ciênc Plur 2017; 3(3):69-80.

5. Rodrigues ET, Foster AC, Santos LL, Ferreira JBB, Falk JW, Fabbro AL. Perfil e trajetória profissional dos egressos da residência em Medicina de Família e Comunidade do estado de São Paulo. Rev Bras Educ Med 2017;41(4):604-14.

6. Ruiz DG, Farenzena GJ, Haeffner LSB. Internato regional e formação médica: percepção da primeira turma pós-reforma curricular. Rev Bras Educ Med 2010;34(1):21-7.

7. Ferreira RC, Silva RF, Aguera CB. Formação do profissional médico: a aprendizagem na atenção básica de saúde. Rev Bras Educ Med 2007;31(1):52-9.

8. Brasil. Ministério da Educação. Conselho Nacional de Educação. Câmara de Educação Superior. Resolução no 3, de 20 de junho de 2014. Diretrizes Curriculares Nacionais do Curso de Graduação em Medicina e outras providências. Brasília: Ministério da Educação; 2014.

9. Silvestre HF, Tesser CD, Ros MA. Avaliação discente de um internato médico em atenção primária à saúde. Rev Bras Educ Med 2016;40(3):383-92.

10. Campos CEA. Os princípios da medicina de família e comunidade. Revista APS 2005. 8(2): 181-190.

11. Gardenal RVC, Marques AMC, Martello LB, Braga LPM, Bonilha $\mathrm{MM}$, Braga $\mathrm{O}$ et al. Estágio supervisionado regional: visão do aluno. Rev Bras Educ Med 2011;35(4):574-77.

12. Bezerra DF, Adami F, Reato LFN, Akerman M. A dor e a delícia do internato de atenção primária em saúde: desafios e tensões. ABCS Health Sci 2015;40 (3):164-70.

13. Souza CFT, Oliveira DLL, Monteiro GS, Barboza HMM, Ricardo GP, Lacerda Neto MC et al. A atenção primária na formação médica: a experiência de uma turma de medicina. Rev Bras Educ Med 2013;37(3):448-54.

14. Google. About Google Forms [acesso em 10 agosto 2018]. Disponível em: https://www.google.com/intl/pt-BR/forms/about/.

15. Gomes NN. Avaliação da formação de profissionais médicos em uma universidade pública em Fortaleza-CE. Fortaleza. Mestrado [Dissertação em Saúde Coletiva] - Universidade Estadual do Ceará; 2014.

16. Bardin L. Análise de conteúdo. São Paulo: Edições 70; 2011.

17. Campos MAF, Forster AC. Percepção e avaliação dos alunos do curso de medicina de uma escola médica pública sobre a importância do estágio em saúde da família na sua formação. Rev Bras Educ Med 2008;32(1):83-9.

REVISTA BRASILEIRA DE EDUCAÇÃO MÉDICA

7 44 (1) : e006; 2020 
18. Soares RS, Oliveira FP, Melo Neto AJ de, Barreto DS, Carvalho ALB, Sampaio J et al. Residência em medicina de família e comunidade: construindo redes de aprendizagens no SUS. Rev Bras Med Fam Comunidade 2018;13(40):1-8.

19. Pinto LLS, Formigli VLA, Rêgo RCF. A dor e a delícia de aprender com o SUS: integração ensino-serviço na percepção dos internos em medicina social. Rev Baiana Saúde Pública 2007;31(1):15-33.

20. Chaves ITS, Grosseman S. O Internato médico e suas perspectivas: estudo de caso com educadores e educandos. Rev Bras Educ Med 2007;31(3): 212-22.

21. Pontes ODA, Sousa-muñoz RL. O internato médico no novo currículo de uma universidade pública: a apreciação do estudante. Rev Bras Educ Med 2014;38(4):519-31.

22. Lima SML, Portela MG, Koster I, Escosteguy CC, Ferreira VMB, Brito C et al. Utilização de diretrizes clínicas e resultados na atenção básica à hipertensão arterial. Cad Saúde Pública 2009;25(9):2001-11.

23. Barría-Pailaquilen RM. Practice based on evidence: an opportunity for quality care. Invest Educ Enferm 2013;31(2):181-2.

24. Duncan BB, Schmidt MI, Falavigna M. Prática da medicina ambulatorial baseada em evidências. In: Duncan BB. Medicina ambulatorial: condutas de atenção primária baseadas em evidências. 4. ed. Porto Alegre: Artmed; 2013. p. 44-66.

25. Schneider LR, Pereira RPG, Ferraz L. A prática baseada em evidência no contexto da atenção primária à saúde. Saúde em Debate. 2018;42(118):594-605.
26. Blank D. A propósito de cenários e atores: de que peça estamos falando? Uma luz diferente sobre o cenário da prática dos médicos em formação. Rev Bras Educ Med 2006;30(1):27-31.

27. Zanolli MB. Metodologias ativas de ensino-aprendizagem na área clínica. In: Marins JJN, Rego S, Lampert JB, Araújo JGC, organizadores. Educação médica em transformação: instrumentos para a construção de novas realidades. São Paulo: Hucitec, ABEM; 2004. p. 40-61.

\section{CONTRIBUIÇÃO DOS AUTORES}

Ivana Cristina Vieira de Lima, Beatriz Yumi Rodrigues Shibuya, Maria das Graças Barbosa Peixoto: concepção, desenho do estudo, análise, interpretação dos dados e redação do artigo.

Leilson Lira de Lima, Paulo Sávio Fontenele Magalhães: concepção, desenho do estudo e redação do artigo.

\section{CONFLITO DE INTERESSES}

Não há conflito de interesses.

\section{ENDEREÇO PARA CORRESPONDÊNCIA}

Ivana Cristina Vieira de Lima

Universidade Estadual do Ceará - UECE. Av. Dr. Silas Munguba, 1700, Itaperi, CEP 60.714-903, Fortaleza - CE, ivana.maia@uece.br 